\title{
Multienergy operation analysis in a superconducting linac based on off-frequency detune method
}

\author{
Zhen Zhang $\odot$, Yuantao Ding, Chris Adolphsen $\odot$, and Tor Raubenheimer \\ SLAC National Accelerator Laboratory, Menlo Park, California 94025, USA
}

(Received 8 August 2019; published 5 November 2019)

\begin{abstract}
The free-electron laser facilities driven by a superconducting radio-frequency (SRF) linac provide highrepetition-rate electron beam, which makes it feasible to feed multiple undulator lines at the same time. In this paper, we study a method of controlling the energy of multiple electron bunches by off-frequency detuning in the SRF linac. Based on the theoretical analysis, we present the available discrete frequency detunes and the optimal linac energy allocation solutions with given energy overhead and periodic energy pattern. The required off-frequency detune is not larger than half of the beam repetition rate, which can be realized with the frequency tuner in SRF cavities. We adopt the configuration of the high energy upgrade of the Linac Coherent Light Source II (LCLS-II-HE) as an example to discuss the possible schemes in practice to support two undulator lines simultaneously. We also discuss the energy jitter increase due to the off-crest acceleration, the tunable energy range with fixed total SRF linac energy capacity, the beam energy chirp and lattice design for multienergy beam transport.
\end{abstract}

DOI: 10.1103/PhysRevAccelBeams.22.110702

\section{INTRODUCTION}

The successful operation of several hard $\mathrm{x}$-ray freeelectron laser (XFEL) facilities [1-4] over the world represents a revolution in the development of light source, which leads to a substantial improvement in the peak brightness by a remarkable 8-10 orders of magnitude [5-7] and opens up vast opportunities for atom and molecule dynamics research at femtosecond scale [8,9]. Most of the present-day XFELs are driven by copper linac with a repetition rate around $100 \mathrm{~Hz}$. This low repetition rate determines only a modest average brightness, similar to that of the third generation storage ring based sources [10]. To support multiple beamlines and deliver xrays with larger energy range, a few schemes have been adopted such as using a kicker and bypass line $[3,4,11]$ and using subharmonic trigger in radio-frequency units [12]. These operation modes further reduces the average brightness due to low repetition rate.

XFELs driven by a superconducting radio-frequency (SRF) linac are capable to deliver megahertz (MHz) electron beams, which greatly enhances the photon average brightness and supports multiple beamlines in parallel and/or cascading configurations [13-17]. With fixed electron beam

\footnotetext{
"zzhang@slac.stanford.edu
}

Published by the American Physical Society under the terms of the Creative Commons Attribution 4.0 International license. Further distribution of this work must maintain attribution to the author(s) and the published article's title, journal citation, and DOI. energy, the photon energies from different beamlines can be varied by adjusting the undulator gap. To further extend the photon energy range, schemes of independently control of the electron beam energy for multiple undulator lines are highly desired, which will greatly impact the design and operation of future SRF based XFEL facilities.

To provide different beam energies for multiple undulator lines from a SRF linac, different schemes can be applied. For example, one can use a similar configuration as in normal-conducting FELs to extract electron beams with a fast kicker and a bypass line, as shown in Fig. 1(a). This scheme is adopted as the baseline design of the present SRF XFEL facilities [13-17]. Since the kicker positions are fixed along the linac, the maximum available beam energy at the kicker position is limited by the capacity of the upstream SRF linac. For example, in the baseline

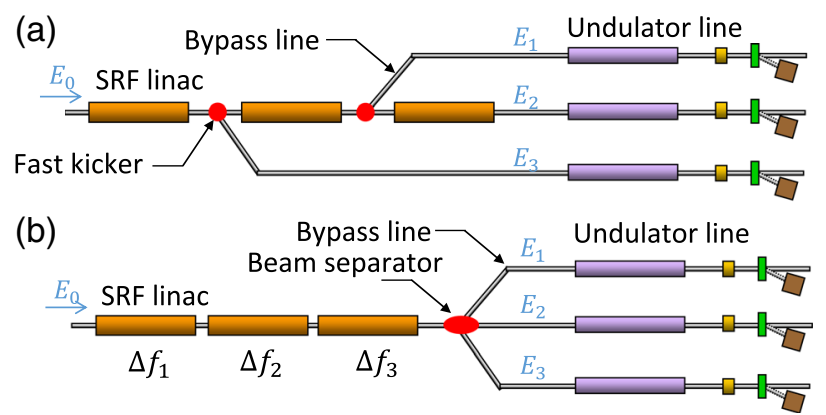

FIG. 1. Two schemes of multi-energy operation of a SRF XFEL: (a) kick the beam to bypass line at desired energy point (b) control the beam energy independently by off-frequency detune of SRF cavities. 
design of the high energy upgrade of the Linac Coherent Light Source II (LCLS-II-HE) [17] at SLAC National Acceleration Laboratory, the electron beam to the soft $\mathrm{X}$-ray (SXR) undulator line is kicked out from the SRF linac at a fixed energy $\sim 3.8 \mathrm{GeV}$ while the maximum energy $8 \mathrm{GeV}$ is sent to the hard $\mathrm{x}$-ray (HXR) line. Any adjustment of the bypass line energy would affect the HXR line energy. Besides, the beam extraction system for $\mathrm{MHz} \mathrm{GeV}$ electron beams requires MHz-triggered submicrosecond highvoltage kickers and strong septum magnets, which make it lengthy and costly to add the extraction bypass beamline to the SRF linac.

There is another scheme for burst-mode superconducting linac based on energy change of bunch trains inside one macropulse, which has been utilized in FLASH2 to support two undulator lines simultaneously [13]. Very recently, another scheme using achromatic and isochronous electron delay system and fast kickers has been proposed to produce multi-energy beams for the SRF linac driven XFELs [18]. The acceleration phases of the electron beams in the last section of the linac are controlled by the customized time delay to achieve different energies.

In this paper, we study a new scheme for multiple-energy operation in a SRF linac based on an off-frequency detune method. This new method was suggested to be one option for LCLS-II-HE [19] and some initial tests are performed at DESY [20]. It is well known that the resonant frequency of a SRF cavity is very sensitive to small mechanical perturbations and can be varied by a frequency tuner which compresses the cavity from the noncoupler side [21,22]. In CW electron beams with fixed time spacing, the detune of nominal resonant frequency of SRF cavities results in different acceleration phases. Combining a series of frequency detunes and optimizing the acceleration phases at nominal resonant frequency, we can produce periodic energy pattern to support multiple undulator lines simultaneously. As shown in Fig. 1(b), the CW electron beams with periodic energy pattern are separated at the downstream of the SRF linac and then sent to the target undulators. The main advantage of this method is the possibility to control the beam energy of each undulator line independently in a wide range (approximately from zero up to the maximum energy capacity of the SRF linac), which can extend the parameter space of the XFEL pulses and improve the facility performance. In addition, the energy difference of the CW beams after the SRF linac makes it possible to adopt the dispersive energy separator based on DC bending magnet to separate and deliver them to different undulator lines. Compared with the pulsed kicker/septum extractor, the bending magnet based separator is simpler and will be a significant saving. For example, when we apply this method to LCLS-II-HE, we can vary the beam energy of the SXR line from 0 to $8 \mathrm{GeV}$ in principle while keeping $8 \mathrm{GeV}$ for the HXR line. In practice, this energy range is limited by the beam transport and beam separator system. However, to achieve these advantages, we have to sacrifice some energy gain of the SRF linac. When the number of undulator lines is larger than two, the electron beams are accelerated at offcrest phases for most energy patterns, resulting in some SRF linac energy overhead compared with the kicker/ extraction method.

This paper is organized as follows. In Sec. II we will analyze the off-frequency detune method theoretically and give the strategy to achieve minimum energy overhead with possible off-frequency detune values. We will present the optimization solution of energy allocation in different cases in Sec. III. The application of this method to LCLS-II-HE and some associated problems will be discussed in Sec. IV. Finally we will give a short summary in Sec. V.

\section{THEORETICAL ANALYSIS}

In this section, we present theoretical analysis of multienergy control in a SRF linac based on the off-frequency detune method. For CW electron beams, we assume the beam repetition rate $f_{r}$ and the time separation of two neighbouring beams $T=1 / f_{r}$. For the nominal resonant frequency of the cavity $f_{c}$ before detuning, all electron beams are accelerated at the same phase $\psi_{c}$, as shown in Fig. 2. When the frequency is detuned by $\Delta f$ and the acceleration phase of the first beam is kept at $\psi_{c}$, the acceleration phase of the $j$ th electron beams is $\psi_{c}+(j-1) \Delta \phi$ with phase difference of two neighboring beams (or we can call it as phase shift)

$$
\Delta \phi=2 \pi T \Delta f .
$$

Larger phase shift corresponds to larger off-frequency detune. In order to reduce the off-frequency detune range, we limit the phase shift within one cycle, i.e., $\Delta \phi \in[-\pi, \pi]$.

To produce energy pattern with period $M$, the acceleration phase of the $(M+1)$ th beam has to be the same with the first one, which can be named as the periodic condition

$$
\psi_{c}+M \Delta \phi=\psi_{c}+2 \pi k
$$

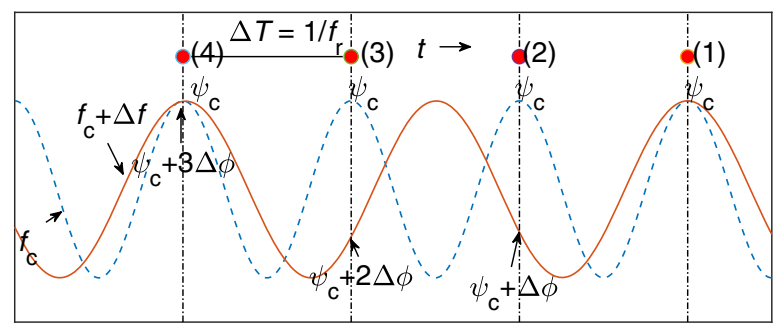

FIG. 2. Acceleration phase changes of the $\mathrm{CW}$ electron beams by the off-frequency detune of the SRF cavities. Here we use $M=3$ and acceleration phase of the fourth beam is the same with the first one. 
where $k$ is an integer. The possible values of the phase shift can be written as

$$
\Delta \phi_{k}=\frac{2 \pi k}{M},
$$

and the available values of $k$ are

$$
k=0, \pm 1, \ldots, \pm(K-1), \pm K,
$$

where $K=\lfloor M / 2\rfloor$ and the function $\lfloor x\rfloor$ returns the largest integer smaller than or equal to $x$. However, in a SRF cavity with acceleration voltage $V_{k}$, initial acceleration phase $\psi_{k}$ before detuning and phase shift $\Delta \phi_{k}$, the energy gain of the $j$ th beam in sequence can be expressed as

$$
\begin{aligned}
\Delta E_{j} & =e V_{k} \cos \left(\psi_{k}+(j-1) \Delta \phi_{k}\right) \\
& =e V_{k} \cos \left(-\psi_{k}+(j-1) \Delta \phi_{-k}\right),
\end{aligned}
$$

with $e$ denoting the electron charge. Equation (5) indicates that the acceleration cavity with initial phase $\psi_{k}$ and phase shift $\Delta \phi_{k}$ can be replaced by the one with $-\psi_{k}$ and $\Delta \phi_{-k}$. In this way, we can further reduce the range of phase shift in half. Without loss of generality, we can let $\Delta \phi \in[0, \pi]$ and the available $k$ is

$$
k=0,1, \ldots,(K-1), K \text {. }
$$

The number of available values of $\Delta \phi$ is $K+1$ and the corresponding off-frequency detune can be expressed by the beam repetition rate $f_{r}$

$$
\Delta f=\frac{k}{M} f_{r}
$$

To cover all phase shifts, the full tunable range of the offfrequency detune is

$$
\Delta f_{\max }= \begin{cases}\frac{1}{2} f_{r}, & M \text { is an even number } \\ \frac{1}{2}\left(1-\frac{1}{M}\right) f_{r}, & M \text { is an odd number }\end{cases}
$$

When the energy pattern period is an odd number, the required off-frequency detune range is less than the half of beam repetition rate. For example, the off-frequency detune range of $M=3$ is $f_{r} / 3$, smaller than the cases of $M=2$ or other numbers.

Using the SRF linac with off-frequency detune to feed multiple undulator lines, we needs to divide the whole linac into $S$ sections, each of which can be described by three variables: maximum energy gain $e V_{s}>0$, initial acceleration phase before detuning $\psi_{s} \in(-\pi, \pi]$ and phase shift $\Delta \phi_{s} \in[0, \pi], s=0,1, \ldots, S-1$. The energy gain of the $j$ th beam can be written as

$$
E_{j}-E_{0}=\sum_{s=0}^{S-1} e V_{s} \cos \left(\psi_{s}+(j-1) \Delta \phi_{s}\right)
$$

where $E_{0}$ is the initial beam energy before the detuned $\mathrm{SRF}$ linac, and $E_{j}$ is the beam energy of the $j$ th electron beam assumed to be sent to the $j$ th undulator line, with $j=1,2, \ldots, M$. Equation (9) can be written in matrix form as

$$
\Phi \mathcal{V}=\mathcal{E}-E_{0}
$$

with $\Phi \in \mathbb{R}^{M \times S}, \quad \Phi(j, s)=\cos \left(\psi_{s}+(j-1) \Delta \phi_{s}\right)$, $\mathcal{V}=\left[e V_{1}, \ldots, e V_{S}\right]^{T} \in \mathbb{R}^{S}$, and $\mathcal{E}=\left[E_{1}, \ldots, E_{M}\right]^{T} \in \mathbb{R}^{M}$. The condition of the matrix $\Phi$ depends on the initial acceleration phases before detuning. For a given set of energy pattern, the allocation of SRF linac $\mathcal{V}$ can be solved as a function of $\Phi$ and $\mathcal{E}-E_{0}$. If we do not put constraints on the number of linac sections and their initial acceleration phases, there are infinite solutions which all produce the required beam sequence. In practice, we need to find the optimal solution for a given energy pattern.

In the off-frequency detune method, it is found that the total energy gain of the $S$ linac sections with assuming on-crest acceleration, $E_{G}=\sum_{s=0}^{S-1} e V_{s}$, is usually larger than the maximum achievable energy change of the beams, indicating that we need more SRF cavities to achieve the designed energy of the facility than usual. This linac energy overhead would be the main concern when applying the method to a real machine. In this paper we adopt the linac energy overhead factor $(\eta)$ to evaluate different energy allocation solutions, which can be defined as the ratio between the extra required linac energy and the maximum beam energy of all undulator lines

$$
\eta=\frac{E_{G}+E_{0}}{E_{\max }}-1 \geq 0
$$

where $E_{\max }$ is the largest beam energy among $E_{j}$, $j=1, \ldots, M$. Larger energy overhead factor means that we need to sacrifice more energy gain of the SRF linac. For a give energy pattern, the overhead factor can be optimized by choosing the initial acceleration phases before detuning for these $S$ sections. For a given total energy gain of the SRF linac, the available energy range can be obtained as well by varying the initial acceleration phases.

When $S>K+1$, i.e., the number of linac sections is larger than the number of available phase shifts, there are at least two sections having the same phase shift. Assuming the $s$ th and $t$ th sections have the same phase shift $\Delta \phi$, the energy gain of the $j$ th beam in sequence is

$$
\begin{aligned}
\Delta E_{j}= & e V_{s} \cos \left(\psi_{s}+(j-1) \Delta \phi\right) \\
& +e V_{t} \cos \left(\psi_{t}+(j-1) \Delta \phi\right) \\
= & e V_{s t} \cos \left(\psi_{s t}+(j-1) \Delta \phi\right),
\end{aligned}
$$


with $V_{s t}, \psi_{s t}$ being the maximum acceleration voltage and initial acceleration phase of the new combined section. The acceleration voltage $V_{s t}$ can be solved as

$$
\begin{aligned}
V_{s t} & =\sqrt{V_{s}^{2}+V_{t}^{2}+2 V_{s} V_{t} \cos \left(\psi_{s}-\psi_{t}\right)}, \\
& \leq V_{s}+V_{t} .
\end{aligned}
$$

The equality can be achieved only when $\psi_{s}=\psi_{t}$. So the linac sections with the same phase shift but different initial acceleration phases can be combined into one section to obtain smaller energy overhead factor.

In the following discussions, we will let $S=K+1$, i.e., each linac section has its unique phase shift. The solutions of the SRF linac energy allocation with lowest overhead factor will be presented for different operated undulator lines.

\section{OPTIMAL SOLUTIONS OF THE OFF-FREQUENCY DETUNE METHOD}

In this section we present the optimal solutions with lowest energy overhead factor of the off-frequency detune method for two, three, and any number of undulator lines, respectively.

\section{A. Two undulator lines}

We first start from the simplest case which has two undulator lines, $M=2$. According to Eq. (6), $K=1$. The possible phase shifts and the corresponding off-frequency detunes from Eqs. (3) and (7) are

$$
\begin{array}{ll}
\Delta \phi_{0}=0, & \Delta f_{0}=0 . \\
\Delta \phi_{1}=\pi, & \Delta f_{1}=f_{r} / 2 .
\end{array}
$$

The SRF linac will be divided into two sections $\left(S_{0}\right.$ and $\left.S_{1}\right)$ as shown in Fig. 3. The initial phases before detuning of the two sections are chosen to minimize the energy overhead factor.

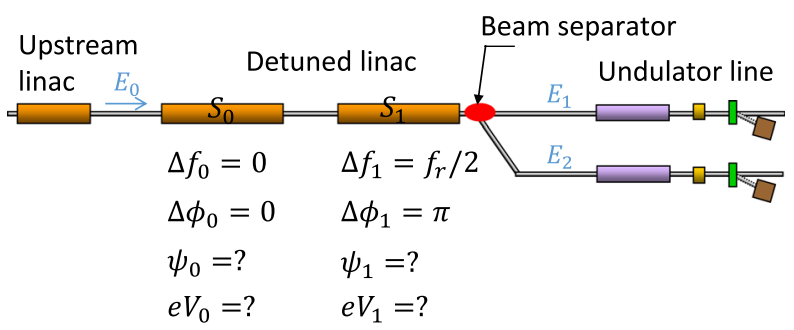

FIG. 3. Layout of the SRF linac to support two undulator lines with off-frequency detune method.
The energy gain of the beam sequence can be written as

$$
\begin{aligned}
& e V_{0} \cos \left(\psi_{0}\right)+e V_{1} \cos \left(\psi_{1}\right)=E_{1}-E_{0}, \\
& e V_{0} \cos \left(\psi_{0}\right)-e V_{1} \cos \left(\psi_{1}\right)=E_{2}-E_{0} .
\end{aligned}
$$

The minimum energy overhead factor $(\eta=0)$ can be achieved by setting $\psi_{0}=\psi_{1}=0$ and the energy gain of each section can be solved with the assumption of $E_{1} \geq E_{2}$

$$
\begin{aligned}
& e V_{0}=\frac{E_{1}+E_{2}}{2}-E_{0}, \\
& e V_{1}=\frac{E_{1}-E_{2}}{2} .
\end{aligned}
$$

When $M=2$, the linac energy overhead is always zero for any energies. For example, to produce 8 and $4 \mathrm{GeV}$ from initial beam energy $4 \mathrm{GeV}$, the total energy gain of the linac is $4 \mathrm{GeV}$ with $2 \mathrm{GeV}$ each section. The SRF cavities in the second section is detuned by $f_{r} / 2$ to accelerate the first beam while decelerating the second one.

\section{B. Three undulator lines}

Following the same analysis as we did above, for three undulator lines $(M=3), K=\lfloor 3 / 2\rfloor=1$. Now the available phase shifts are $\Delta \phi_{0}=0$, and $\Delta \phi_{1}=2 \pi / 3$. The corresponding off-frequency detune are $\Delta f_{0}=0$ and $\Delta f_{1}=f_{r} / 3$. We illustrate the layout of the SRF linac in Fig. 4. In this case, the energy gain of the three undulator lines can be expressed as

$$
\begin{aligned}
e V_{0}+e V_{1} \cos \left(\psi_{1}\right) & =E_{1}-E_{0}, \\
e V_{0}+e V_{1} \cos \left(\psi_{1}+\frac{2 \pi}{3}\right) & =E_{2}-E_{0}, \\
e V_{0}+e V_{1} \cos \left(\psi_{1}+\frac{4 \pi}{3}\right) & =E_{3}-E_{0} .
\end{aligned}
$$

Here we already let the initial acceleration phase of the cavity without off-frequency detune $\left(\Delta f_{0}=0\right)$ be zero $\left(\psi_{0}=0\right)$ to reduce the energy overhead.

Without loss of generality, we can assume that $E_{1}$ is the largest beam energy in the three lines, i.e., $E_{1} \geq\left(E_{2}, E_{3}\right)$.

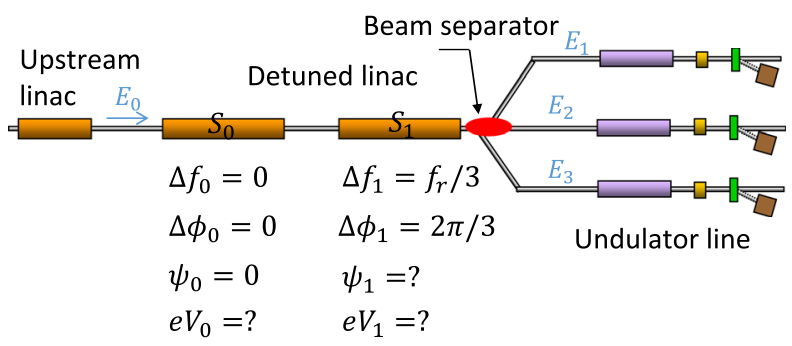

FIG. 4. Layout of the SRF linac to support three undulator lines with off-frequency detune method. 
The initial phase of the detuned section $\left(\psi_{1}\right)$ and energy gain of the two sections can be solved as

$$
\begin{aligned}
\psi_{1} & =\arctan \left(\frac{\sqrt{3}\left(E_{3}-E_{2}\right)}{2 E_{1}-E_{2}-E_{3}}\right) \\
e V_{0} & =\frac{1}{3}\left(E_{1}+E_{2}+E_{3}-3 E_{0}\right) \\
e V_{1} & =\frac{2}{3}\left(E_{1}^{2}+E_{2}^{2}+E_{3}^{2}-E_{1} E_{2}-E_{1} E_{3}-E_{2} E_{3}\right)^{1 / 2} .
\end{aligned}
$$

The energy overhead factor can be calculated by

$$
\eta=\frac{e V_{0}+e V_{1}+E_{0}}{E_{1}}-1
$$

In Fig. 5, we present the linac energy overhead factor for different energy patterns with $E_{0}=E_{1} / 2$. The other two energies are also scaled with $E_{1}$. It can be seen that small energy overhead factor appears along the diagonal area where the other two beam energies are close. At the top-left and bottom-right corners where the two energies have large difference, the energy overhead factor can reach up to $\sim 30 \%$. However, in this case, one of the two energies approaches 0 , which is not the region of interest in most facilities. When we limit the energy overhead factor less than $10 \%$, we can cover most of the energy requirements for the other two undulator lines. Here we give some specific examples. When the energy requirement of the three undulator lines is $8-6-4 \mathrm{GeV}$ from initial energy $E_{0}=4 \mathrm{GeV}$, according to Eqs. (18), the first section without off-frequency detune accelerates beams on-crest by $2 \mathrm{GeV}$. The on-crest energy gain of the second section with frequency detune $f_{r} / 3$ is $2.31 \mathrm{GeV}$. The acceleration phases of the three beams are $-30^{\circ}, 90^{\circ}$, and $210^{\circ}$, respectively. The total energy gain of the SRF linac is $4.31 \mathrm{GeV}$ with energy overhead factor $\eta=(2+2.31+$ 4) $/ 8-1 \approx 3.9 \%$. When $E_{2}=E_{3}$, the initial phase of the detuned section $\psi_{1}=0$ and the energy overhead factor

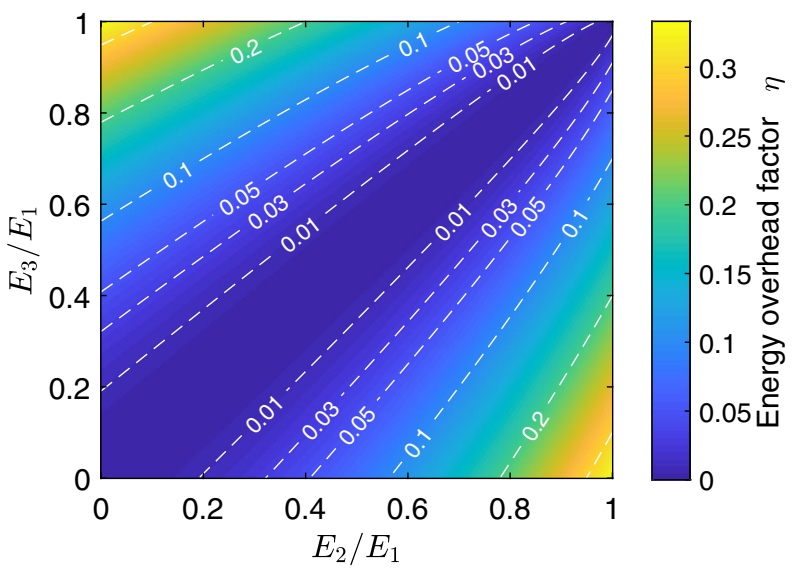

FIG. 5. Energy overhead factor for different beam energy combinations of the other two undulator lines with the condition of $E_{0}=E_{1} / 2$.

$\eta=0$. For example, to produce $8-6-6 \mathrm{GeV}$ from initial $4 \mathrm{GeV}$, the total $4 \mathrm{GeV}$ energy gain of the linac is divided to $e V_{0}=8 / 3 \mathrm{GeV}$ and $e V_{1}=4 / 3 \mathrm{GeV}$, and the second section is detuned with frequency offset $f_{r} / 3$.

Note that the off-frequency detune range of $M=3$ is smaller than the one of $M=2$. In practice we can feed two undulator lines with energy pattern of $M=3$. For example, in the energy pattern of $8-6-6 \mathrm{GeV}$, the first beam $(8 \mathrm{GeV})$ is kicked to one undulator line while the other two $(6 \mathrm{GeV})$ are transported to the second. The beam repetition rate of the first undulator line is half of the second. When we apply this configuration to the LCLS-II-HE $1 \mathrm{MHz}$ beam, the full range of the off-frequency detune is $333 \mathrm{kHz}$.

\section{General optimal solutions}

In this subsection, we consider the general optimal solutions of the off-frequency detune method. For energy pattern period $M$, the whole SRF linac is divided into $K+1$ sections $(k=0,1, \ldots, K)$ and the energy gain of $M$ beams can be written as

$$
\begin{aligned}
& e V_{0} \cos \left(\psi_{0}\right)+e V_{1} \cos \left(\psi_{1}\right)+\cdots+e V_{K} \cos \left(\psi_{K}\right)=\Delta E_{1}, \\
& e V_{0} \cos \left(\psi_{0}\right)+e V_{1} \cos \left(\psi_{1}+\frac{2 \pi(j-1)}{M}\right)+\cdots+e V_{K} \cos \left(\psi_{K}+\frac{2 \pi K(j-1)}{M}\right)=\Delta E_{j}, \\
& \begin{array}{lll}
\ldots & \ldots & \ldots
\end{array} \\
& e V_{0} \cos \left(\psi_{0}\right)+e V_{1} \cos \left(\psi_{1}+\frac{2 \pi(M-1)}{M}\right)+\cdots+e V_{K} \cos \left(\psi_{K}+\frac{2 \pi K(M-1)}{M}\right)=\Delta E_{M} .
\end{aligned}
$$

Here $\Delta E_{j}=E_{j}-E_{0}$ means the energy change of the $j$ th beam from the initial beam energy $E_{0}$. For $k=0$, it can be solved by summing over all equations as 


$$
e V_{0} \cos \left(\psi_{0}\right)=\frac{1}{M} \sum_{j=1}^{M} \Delta E_{j} .
$$

We can let $\psi_{0}=0$ to reduce the energy overhead and then the on-crest energy gain without frequency detune can be written as

$$
e V_{0}=\frac{1}{M} \sum_{j=1}^{M} \Delta E_{j}
$$

We can combine this section and the initial beam energy together

$$
E_{0}+e V_{0}=\frac{1}{M} \sum_{j=1}^{M} E_{j},
$$

which means that the average energy of all beams determines the start of off-frequency detune.

When $k>0$ and $2 k \neq M$, replacing $\cos (\theta)$ with $\frac{1}{2}\left(e^{i \theta}+e^{-i \theta}\right)$ and summing over all equations after multiplying a factor of $e^{ \pm i(2 \pi k(j-1) / M)}$, we can obtain

$$
e V_{k} M e^{ \pm i \psi_{k}}=2 \sum_{j=1}^{M} \Delta E_{j} e^{\mp i \frac{2 \pi k(j-1)}{M}} .
$$

The initial acceleration phase $\psi_{k}$ can be expressed as

$$
\begin{aligned}
\psi_{k}= & \arctan 2\left\{-\sum_{j=1}^{M} \Delta E_{j} \sin \left[\frac{2 \pi k(j-1)}{M}\right],\right. \\
& \left.\times \sum_{j=1}^{M} \Delta E_{j} \cos \left[\frac{2 \pi k(j-1)}{M}\right]\right\},
\end{aligned}
$$

where $\arctan 2(y, x)$ is the four-quadrant inverse tangent function. The maximum energy gain of each section $e V_{k}$ can be obtained as

$$
\begin{aligned}
e V_{k}= & \frac{2}{M}\left(\left\{\sum_{j=1}^{M} \Delta E_{j} \sin \left[\frac{2 \pi k(j-1)}{M}\right]\right\}^{2}\right. \\
& \left.+\left\{\sum_{j=1}^{M} \Delta E_{j} \cos \left[\frac{2 \pi k(j-1)}{M}\right]\right\}^{2}\right)^{1 / 2} .
\end{aligned}
$$

When $2 k=M$, i.e., $M$ is an even number and we consider the section with phase shift $\Delta \phi_{K}=\pi$, multiplying $e^{i \pi(j-1)}$ to each equation and summing over all equations, we can get

$$
e V_{K} \cos \left(\psi_{K}\right)=\frac{1}{M} \sum_{j=1}^{M} \Delta E_{j} e^{i \pi(j-1)} .
$$

Similarly with the case of $k=0$, we can let $\psi_{K}=0$ or $\psi_{K}=\pi$ to reduce energy overhead. So the on-crest energy gain of the section with $f_{r} / 2$ detune $(K=M / 2)$ is

$$
e V_{K}=\frac{1}{M}\left|\sum_{j=1}^{M} \Delta E_{j} e^{i \pi(j-1)}\right| .
$$

The energy overhead factor can be calculated by the definition in Eq. (11). If we assume that $E_{1}$ is the largest energy in the beam sequence, the energy overhead factor $\eta$ will be larger than 0 when any $\psi_{k}$ is not zero. In this case, the condition to achieve zero energy overhead is

$$
\begin{aligned}
& \sum_{j=1}^{M} \Delta E_{j} \sin \left[\frac{2 \pi k(j-1)}{M}\right]=0, \\
& \sum_{j=1}^{M} \Delta E_{j} \cos \left[\frac{2 \pi k(j-1)}{M}\right]>0 .
\end{aligned}
$$

for all $k=0,1, \ldots, K$. The first equation in Eq. (29) can be further simplified. When $M$ is an even number, it can be expressed as $E_{j}=E_{M-j+1}$ for $j=2, \ldots, M-1$. When $M$ is an odd number, the simplified form is $E_{j}=E_{M-j+2}$ for $j=2, \ldots, M$. However, for beam energies which meet the first equation, we also need to check the validity of the second inequality. The condition for $\eta=0$ is satisfied by any energy pattern at $M=2$ and $E_{2}=E_{3}, E_{1} \geq\left(E_{2}, E_{3}\right)$ at $M=3$.

Here we give some examples to apply the general optimal solutions. For five undulator lines $(M=5)$, $K=\lfloor 5 / 2\rfloor=2$ and the whole linac is divided into $3 \mathrm{sec}-$ tions. One necessary condition for $\eta=0$ is $E_{2}=E_{5}$ and $E_{3}=E_{4}$. Assuming initial energy $E_{0}=4 \mathrm{GeV}$ and the desired energies are $8 / 7 / 6 / 6 / 7 \mathrm{GeV}$, all initial acceleration phases before detuning are zero and the energy allocation plan is $2.80 / 1.05 / 0.15 \mathrm{GeV}$. However, when the desired energies are $8 / 7 / 5 / 5 / 7 \mathrm{GeV}$, the initial acceleration phase of the third section is $-180^{\circ}$ and the energy overhead factor $\eta=2.4 \%>0$ though the first equation in Eq. (29) is satisfied.

In addition, the condition for minimum energy overhead can provide a direction for optimization. For example, when the desired energies are $8 / 7.5 / 7 / 6 / 5 \mathrm{GeV}$, the energy overhead factor $\eta=9.9 \%$. If we rearrange the energies as $8 / 7.5 / 6 / 5 / 7 \mathrm{GeV}$ which is closer to the optimal condition, the new energy overhead factor $\eta=6.1 \%$. So for a given set of energies, the energy overhead can be optimized by suitable sorting in beam sequence.

\section{APPLICATION IN THE LCLS-II-HE}

In this section we apply the off-frequency detune method with the beam parameters of the LCLS-II-HE [17]. The LCLS-II-HE is a high energy upgrade of the LCLS-II, 
a superconducting CW XFEL facility at SLAC, which will increase the beam energy from $4 \mathrm{GeV}$ to $8 \mathrm{GeV}$. It will provide ultrafast $\mathrm{x}$-rays from the soft and hard undulator lines at repetition rate up to $1 \mathrm{MHz}$.

A compact frequency tuner has been designed for the LCLS-II project, including slow/coarse tuner and fast/fine tuner. The schematic and test performance of the tuner can be found in Refs. [23,24]. Coarse frequency tuning is achieved with a motor-driven end-lever tuner. When the cavities are cooled to $2 \mathrm{~K}$ and the frequency is set to $1.3 \mathrm{GHz}$, there is typically a $+/-300 \mathrm{kHz}$ range available over which the tuner can change the cavity frequency. With minor modifications to the tuner design, the low end of the tuning range could likely be extended to $-500 \mathrm{kHz}$, which would allow two energy operation at about $1 \mathrm{MHz}$. However multibeam operation can still be achieved with the current tuner design at lower beam repetition rates.

\section{A. Two undulator lines in the LCLS-II-HE}

The present design of the LCLS-II-HE contains two undulator lines, SXR and HXR lines. To support these two lines by the off-frequency method, there are different solutions and each of them will be discussed as follows.

The first one is the standard scheme for two-undulator case. The whole linac is divided into two parts and the offfrequency detune of the second part is half of the beam rates. According to Eq. (16) we can produce any set of beam energies without energy overhead in the last $4 \mathrm{GeV}$ $\mathrm{SRF}$ linac. For $1 \mathrm{MHz}$ beam, the frequency detune is $500 \mathrm{kHz}$, which is out of the current frequency detune range of the SRF cavity in the LCLS-II-HE.

Second, we can use the scheme of $M=3$ to support two undulator lines. The first beam is sent to the HXR undulator line and the other two with the same energy are for the SXR. The beam repetition rates in the two undulator lines are different. As discussed previously, the energy overhead factor is zero for any two energies and the required frequency detune is one third of beam repetition rate, i.e., $333 \mathrm{kHz}$ for $1 \mathrm{MHz}$ beam.

The third method is to use a special solution of the four-line scheme. In four-line scheme, the SRF linac needs to be divided into three sections with off-frequency detune of $0, f_{r} / 4$ and $f_{r} / 2$. For a special energy pattern ( $\left.E_{1}=E_{2}, E_{3}=E_{4}\right)$, the required energy gain of the third section can be zero and the maximum off-frequency detune is only $f_{r} / 4$. The acceleration phases of the four beams in the detuned linac are shown in Fig. 6 and we will obtain two groups of energies. The final two energies can be varied by changing the fraction of detuned SRF linac. With the parameters of the LCLS-II-HE, the available energies of the two undulator lines are shown in Fig. 6. The two energies are correlated with each other and the minimum SXR line energy $(1.18 \mathrm{GeV})$ can be achieved when we detune the last $4 \mathrm{GeV}$ linac by a quarter of beam rate. When the SXR line energy is $3-6 \mathrm{GeV}$, the corresponding HXR line energy is

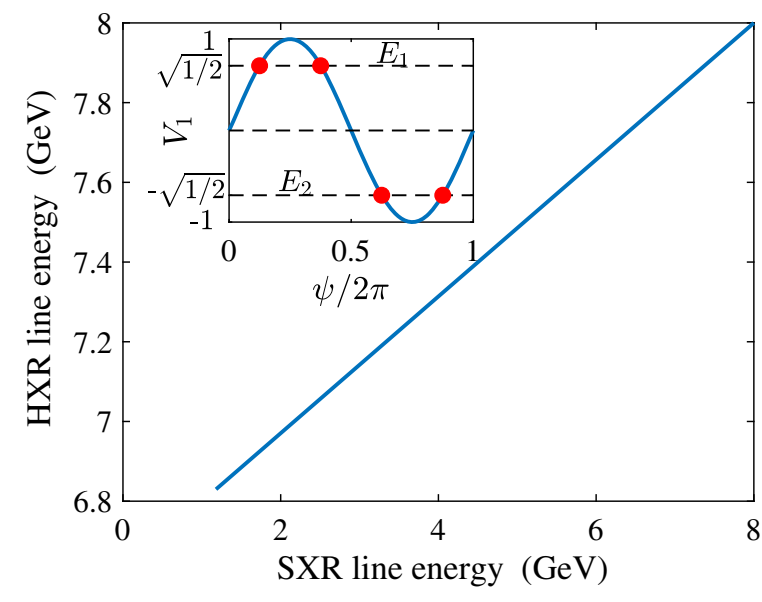

FIG. 6. Available beam energies for SXR and HXR lines when using the special solution of four-line scheme.

7.14-7.65 GeV, which is less than $8 \mathrm{GeV}$. Despite of these limitations, the small off-frequency detune range $(250 \mathrm{kHz}$ for $1 \mathrm{MHz}$ beam) makes it still very promising in practice.

\section{B. Beam energy jitter}

In the off-frequency detune method, electron beams are accelerated at off-crest phases, where the beam energy is more sensitive to the phase jitter compared with the oncrest acceleration. The acceleration phase error of the $\mathrm{CW}$ beams comes from RF phase jitter, cavity frequency error and beam arrival time jitter. Here we study the beam energy jitter resulted from the acceleration phase jitter in the detuned SRF linac section. In the last SRF linac section (L4) of the LCLS-II-HE, there are 27 cryomodules and each of them contains 8 cavities inside. So we will have 216 cavities whose phase and frequency can be varied independently. The number of detuned cavities depends on the target energies of the undulator lines. Assuming the acceleration phase in each cavity follows the normal distribution with mean $\theta$ and standard deviation $\sigma$, the relative standard deviation of energy gain through the cavity can be written as

$$
\sigma_{\delta}=\sqrt{\frac{1}{2}+\frac{1}{2} \cos (2 \theta) e^{-2 \sigma^{2}}-\cos ^{2}(\theta) e^{-\sigma^{2}}} .
$$

The smallest energy spread is achieved at $\theta=0$ or $\pi$, i.e. on-crest acceleration or deceleration. Summing over all cavities with their own phase and amplitude, we can calculate the energy jitter of the beam to SXR line in the three possible schemes above for the LCLS-II-HE, which are shown in Fig. 7. It is assumed that the standard deviation of acceleration phase for each SRF cavity $\sigma=$ $0.01^{\circ}[25,26]$ and all cavities have the same acceleration gradient. The energy range considered in SXR line is $2-6 \mathrm{GeV}$ and the beam energy of HXR line is kept at $8 \mathrm{GeV}$ for the two/three-line schemes. In the two-line scheme, 


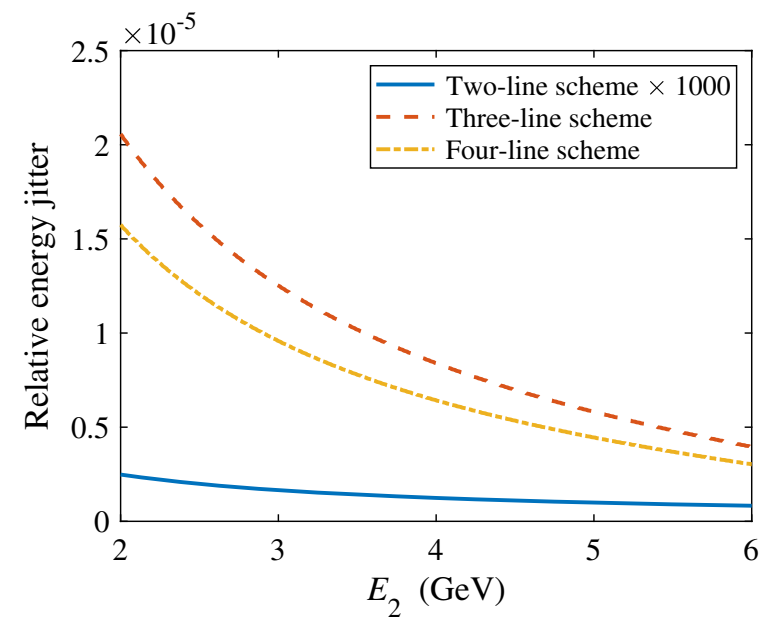

FIG. 7. Relative energy jitter of electron beam to SXR line in the three schemes considered for two undulator lines at the LCLS-II-HE. The standard deviation of acceleration phase jitter is $\sigma=0.01^{\circ}$.

the energy jitter due to the phase jitter in L4 is around $10^{-9}$, which is similar with the regular operation configuration. In the three/four-line schemes, due to their off-crest acceleration phases, the energy jitter can reach $\sim 10^{-5}$ when assuming phase jitter $\sigma=0.01^{\circ}$. This level of energy jitter is still within the requirement of the machine design. However, in a standard configuration, the phase jitter of the last linac section could be relaxed. Here the requirement of $0.01^{\circ}$ is still preferred. More analysis needs to be performed based on the detailed machine parameters.

\section{Tunable energy range with limited total energy gain}

When the energy pattern period is larger than two, the linac energy overhead is usually larger than 0 for a given set of beam energies. In practice, the total energy gain of the SRF linac is always limited by the facility scale. Compared with the minimum energy overhead factor discussed above, the available energy range with limited total energy gain would attract more interests during the application of the method. Here we consider the tunable range when using the LCLS-II-HE linac to support three undulators.

In Fig. 8, we present the tunable energy range (colored area) for different beam energy $E_{1}$ of the first undulator line. The total energy gain of the linac for frequency detune is $E_{G}=4 \mathrm{GeV}$ and the initial beam energy is $E_{0}=4 \mathrm{GeV}$. It can be seen that the tunable range is symmetrical along the diagonal of the energy space. When $E_{1}=6 \mathrm{GeV}$, we can almost cover the full energy range except the area close to the top-left and bottom-right corners. As the increase of $E_{1}$, the flexibility of energy control is getting less and the energy band is becoming narrower gradually. When $E_{1}=7.8 \mathrm{GeV}$, the available range appears around the diagonal. Fixing the second energy $E_{2}=4 \mathrm{GeV}$, the third energy $E_{3}$ can be varied from $2-5.6 \mathrm{GeV}$, which is still be
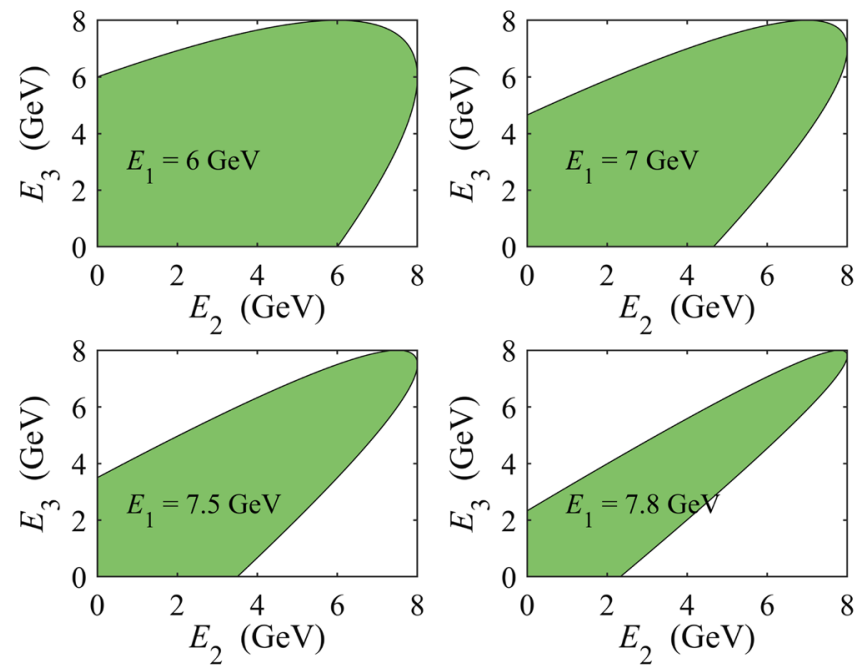

FIG. 8. Tunable energy range of $E_{2}$ and $E_{3}$ (colored area) when the first beam energy $E_{1}=6 / 7 / 7.5 / 7.8 \mathrm{GeV}$, initial beam energy $E_{0}=4 \mathrm{GeV}$ and the on-crest energy gain of the linac for off-frequency detune is limited to be $4 \mathrm{GeV}$.

able to meet the requirements for the beam energy in the soft X-ray line of the LCLS-II-HE.

\section{Beam energy chirp}

In the off-frequency method, the electron beams are accelerated at different phases those are far from the oncrest acceleration, which may lead to different energy chirp in the electron beam. However, the effect of the rf-induced energy chirp is small since the electron bunch length is much shorter than the rf wavelength of the SRF linac. We take the LCLS-II-HE beam as an example. The full length of electron beam is $\sim 200 \mathrm{fs}$, which only occupies $\sim 0.1^{\circ}$ in
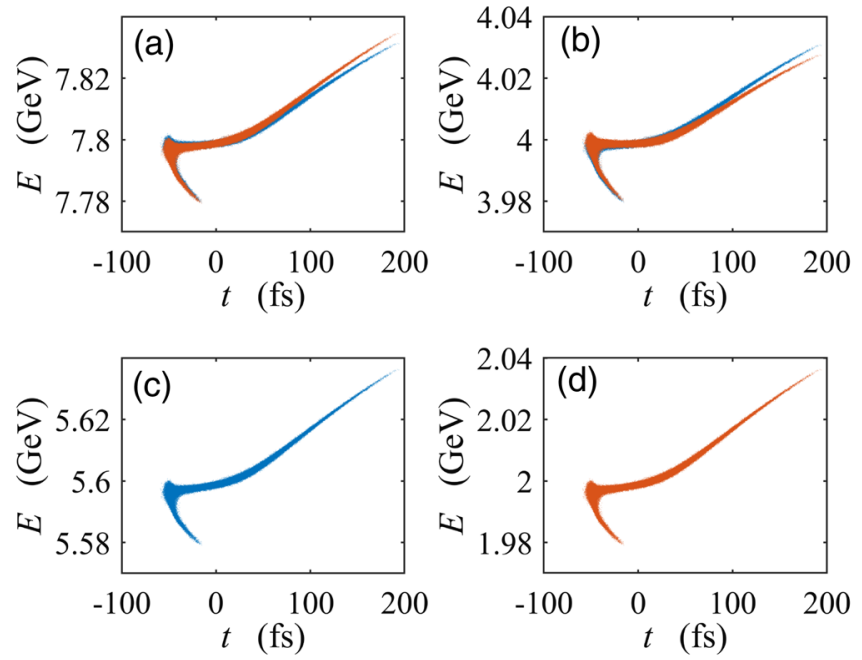

FIG. 9. Longitudinal phase space of the electron beam: (a) the first beam of case A (blue dot) and case B (red dot); (b) the second beam of case A (blue dot) and case B (red dot); (c) the third beam of case $A$; (d) the third beam of case $B$. 
TABLE I. Two cases of SRF linac settings for three-energy operation.

\begin{tabular}{lcc}
\hline \hline Parameter & Case A & Case B \\
\hline Initial energy $E_{0}(\mathrm{GeV})$ & 4.0 & 4.0 \\
1st beam $E_{1}(\mathrm{GeV})$ & 7.8 & 7.8 \\
2nd beam $E_{2}(\mathrm{GeV})$ & 4.0 & 4.0 \\
3rd beam $E_{3}(\mathrm{GeV})$ & 5.6 & 2.0 \\
1st section $e V_{0}(\mathrm{GeV})$ & 1.8 & 0.6 \\
2nd section $e V_{1}(\mathrm{GeV})$ & 2.2 & 3.4 \\
2nd section $\psi_{1}\left({ }^{\circ}\right)$ & 24.79 & -19.84 \\
1nd section $\Delta f_{0}\left(f_{r}\right)$ & 0 & 0 \\
2nd section $\Delta f_{1}\left(f_{r}\right)$ & $1 / 3$ & $1 / 3$ \\
\hline \hline
\end{tabular}

the $1.3 \mathrm{GHz}$ SRF cavity. We compare the longitudinal phase space of electron beam for three-energy operation scheme in Fig. 9, whose SRF linac settings are shown in Table I. In these two cases, the first two beams both have the same energy $(7.8$ and $4.0 \mathrm{GeV})$. And the third beam is $5.6 \mathrm{GeV}$ in case $\mathrm{A}$ and $2.0 \mathrm{GeV}$ in case $\mathrm{B}$. The phase space is simulated by Elegant $[27,28]$ with beam collective effects. It can be seen that all beams have very similar phase space. There is small difference between the two cases at the tail of the first and second beams, which can be neglected for FEL lasing.

\section{E. Lattice design for multi-energy operation}

In the multienergy operation, the lattice design in the detuned linac and the downstream transport line is one of the most critical problems. As the increase of energy difference along the SRF linac, the focusing strength from quadrupoles on the beam varies gradually, resulting in the deviation from the designed lattice. To transport the multienergy beams, we can adopt moderate focusing and reasonable $\beta$ function for the median energy of the tunable
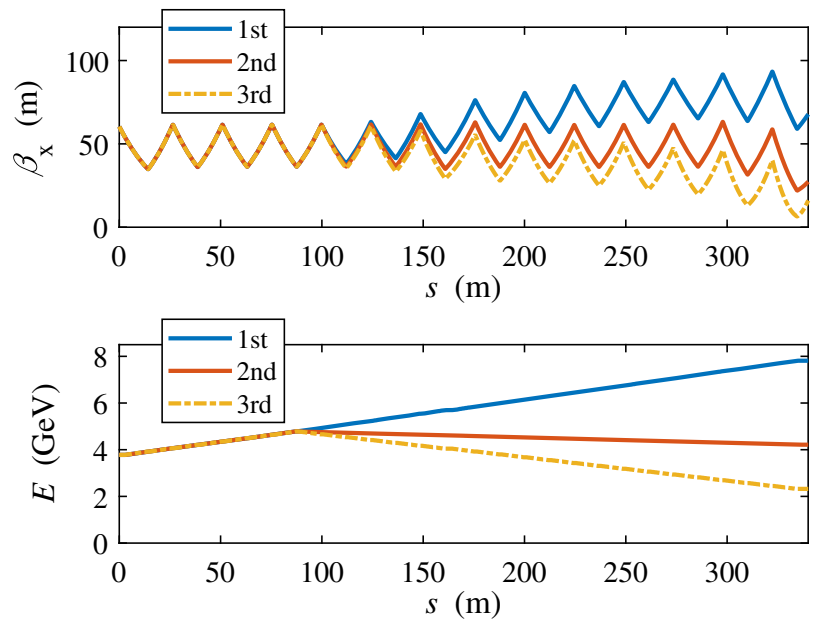

FIG. 10. The $\beta_{x}$ function (top) and energy evolution (bottom) in the detuned linac of the three electron beams of case B in Table. I in the LCLS-II-HE. range. In Fig. 10, we present the $\beta$ functions in the detuned linac of the three electron beams of case B in Table I. We adopt the design lattice of the LCLS-II-HE to the second beam $(4 \mathrm{GeV})$ and all quadrupoles are scaled with beam energy for the other two beams. For the first beam with high energy, the weaker focusing gradually results in larger $\beta$ function while for the third beam with low energy the stronger focusing leads to smaller values. The simulations in Fig. 10 demonstrate that we can propagate the multienergy beams through the $4 \mathrm{GeV}$ SRF linac by carefully choosing the focusing strength. After the linac, the beams can share some beam transport line before they are separated by beam separator. A dedicated group of matching quadrupoles can be used to match the beam to the required transverse size of each line. In addition to the quadruples, the bending angle of the dipole also depends on the beam energy, which could be used to separate the multienergy beams. Details of the lattice design for this multienergy operation scheme will be studied separately.

\section{SUMMARY}

In this paper, we have performed theoretical analysis of the off-frequency detune method in SRF cavities, which is considered to produce multienergy $\mathrm{CW}$ electron beams for multiple undulator lines. We explore the features and limitations of this method, and reveal the requirements for machine parameters. For a given energy pattern period, the possible off-frequency detune values are limited by the periodic condition. The required off-frequency detune range is not larger than half of the beam repetition rate. After simplifying the parameters in application, we present the optimal solutions of linac energy allocation to achieve the minimum energy overhead for any given energy pattern. For the two undulator lines in the LCLSII-HE, we discuss three possible schemes to apply the offfrequency detune method, which can be chosen based on the available frequency detune range. The beam energy jitter induced by the phase jitter of the detuned SRF linac is still within the requirement of machine design. The energy chirp induced by off-crest acceleration is very small and can be neglected for FEL lasing. The lattice of the linac and the downstream beam line needs to be optimized to control the beam size for multienergy beams.

\section{ACKNOWLEDGMENTS}

We thank M. Guetg for helpful discussion. This work was supported by U.S. Department of Energy Contracts No. DE-AC02-76SF00515.

[1] P. Emma, R. Akre, J. Arthur, R. Bionta, C. Bostedt, J. Bozek, A. Brachmann, P. Bucksbaum, R. Coffee, F-J. Decker et al., First lasing and operation of an ångstrom-wavelength free-electron laser, Nat. Photonics 4, 641 (2010). 
[2] T. Ishikawa, H. Aoyagi, T. Asaka, Y. Asano, N. Azumi, T. Bizen, H. Ego, K. Fukami, T. Fukui, Y. Furukawa et al., A compact $\mathrm{X}$-ray free-electron laser emitting in the subangstrom region, Nat. Photonics 6, 540 (2012).

[3] H.-S. Kang, C.-K. Min, H. Heo, C. Kim, H. Yang, G. Kim, I. Nam, S.-Y. Baek, H.-J. Choi, G. Mun et al., Hard X-ray free-electron laser with femtosecond-scale timing jitter, Nat. Photonics 11, 708 (2017).

[4] C. Milne, T. Schietinger, M. Aiba, A. Alarcon, J. Alex, A. Anghel, V. Arsov, C. Beard, P. Beaud, S. Bettoni et al., SwissFEL: The swiss X-ray free electron laser, Appl. Sci. 7, 720 (2017).

[5] Z. Huang and K.-J. Kim, Review of x-ray freeelectron laser theory, Phys. Rev. Accel. Beams 10, 034801 (2007).

[6] L. B. Fletcher, H. J. Lee, T. Döppner, E. Galtier, B. Nagler, P. Heimann, C. Fortmann, S. LePape, T. Ma, M. Millot et al., Ultrabright $\mathrm{x}$-ray laser scattering for dynamic warm dense matter physics, Nat. Photonics 9, 274 (2015).

[7] C. Pellegrini, A. Marinelli, and S. Reiche, The physics of X-ray free-electron lasers, Rev. Mod. Phys. 88, 015006 (2016).

[8] H. N. Chapman, P. Fromme, A. Barty, T. A. White, R. A. Kirian, A. Aquila, M. S. Hunter, J. Schulz, D. P. DePonte, U. Weierstall et al., Femtosecond x-ray protein nanocrystallography, Nature (London) 470, 73 (2011).

[9] C. Bostedt, S. Boutet, D. M. Fritz, Z. Huang, H. J. Lee, H. T. Lemke, A. Robert, W. F. Schlotter, J. J. Turner, and G. J. Williams, Linac coherent light source: The first five years, Rev. Mod. Phys. 88, 015007 (2016).

[10] K.-J. Kim, Z. Huang, and R. Lindberg, Synchrotron Radiation and Free-Electron Lasers (Cambridge University Press, Cambridge, England, 2017).

[11] E. Allaria, L. Badano, S. Bassanese, F. Capotondi, D. Castronovo, P. Cinquegrana, M. B. Danailov, G. D. Auria, A. Demidovich, R. D. Monte et al., The fermi free-electron lasers, J. Synchrotron Radiat. 22, 485 (2015).

[12] T. Hara, K. Tamasaku, T. Asaka, T. Inagaki, Y. Inubushi, T. Katayama, C. Kondo, H. Maesaka, S. Matsubara, T. Ohshima et al., Time-interleaved multienergy acceleration for an X-ray free-electron laser facility, Phys. Rev. Accel. Beams 16, 080701 (2013).

[13] B. Faatz, E. Plönjes, S. Ackermann, A. Agababyan, V. Asgekar, V. Ayvazyan, S. Baark, N. Baboi, V. Balandin, N. von Bargen et al., Simultaneous operation of two soft x-ray free-electron lasers driven by one linear accelerator, New J. Phys. 18, 062002 (2016).

[14] M. Altarelli, R. Brinkmann, M. Chergui, W. Decking, B. Dobson, S. Düsterer, G. Grübel, W. Graeff, H. Graafsma, J. Hajdu et al., The european X-ray free-electron laser, DESY Technical design report No. 97, 2006, p. 1-26.

[15] T. Raubenheimer et al., LCLS-II final design report. Technical report, SLAC Technical Report. No. LCLSII1.1-DR-0251-R0, 2015.
[16] Z. T. Zhao, D. Wang, Z. H. Yang, and L. Yin, SCLF: An 8gev cw scrf linac-based x-ray fel facility in shanghai, in Proceeding of 38th Int. Free Electron Laser Conf. (FEL'17), Santa Fe, NM, USA, 2017 (JACoW, Geneva, Switzerland, 2018), paper MOP055, pp. 182-184, https:// doi.org/10.18429/JACoW-FEL2017-MOP055.

[17] T. Raubenheimer et al., The LCLS-II-HE, a high energy upgrade of the lcls-ii, in 60th ICFA Advanced Beam Dynamics Workshop on Future Light Sources (FLS'18), Shanghai, China, 5-9 March 2018 (JACOW Publishing, Geneva, Switzerland, 2018), pp. 6-11.

[18] J. Yan and H. Deng, Multi-beam-energy operation for the continuous-wave $\mathrm{X}$-ray free electron laser, Phys. Rev. Accel. Beams 22, 090701 (2019).

[19] J. Galayda (private communication).

[20] E. Vogel (private communication).

[21] M. Liepe, W. D. Moeller, and S. N. Simrock, Dynamic lorentz force compensation with a fast piezoelectric tuner, in PACS2001. Proceedings of the 2001 Particle Accelerator Conference (Cat. No. O1CH37268) (IEEE, New York, 2001), Vol. 2, pp. 1074-1076.

[22] S. Simrock, G. Petrosyan, A. Facco, V. Zviagintsev, S. Andreoli, and R. Paparella, First demonstration of microphonic control of a superconducting cavity with a fast piezoelectric tuner, in Proceedings of the 2003 Particle Accelerator Conference (IEEE, New York, 2003), Vol. 1, pp. 470-472.

[23] Y. Pischalnikov, E. Borissov, I. Gonin, J. Holzbauer, T. Khabiboulline, W. Schappert, S. Smith, and J.-C. Yun, Design and test of the compact tuner for narrow bandwidth SRF cavities, in Proceeding of 6th International Particle Accelerator Conference (IPAC'15), Richmond, VA, USA, 2015 (2015), pp. 3352-3354, https://doi.org/10.18429/ JACoW-IPAC2015-WEPTY035.

[24] J. Holzbauer, W. Schappert, Y. Pischalnikov, and J.-C. Yun, Performance of srf cavity tuners at lcls ii prototype cryomodule at fnal, in Proc. of Linear Accelerator Conference (LINAC'16), East Lansing, MI, USA, 25-30 September 2016 (JACOW, Geneva, Switzerland, 2017).

[25] P. Emma, LCLS-II physics requirement document: Linac requirements, Technical report No. LCLSII-2.4-PR-0041R2, 2016.

[26] L. R. Doolittle et al., High precision rf control for SRF cavities in LCLS-II, in Proc. of International Conference on RF Superconductivity (SRF'17), Lanzhou, China, July 17-21, 2017 (JACoW, Geneva, Switzerland, 2018), Vol. 18, pp. 944-950.

[27] M. Borland, Elegant, Technical report, Advanced Photon Source LS-287, 2003.

[28] Y. Wang and M. Borland, Pelegant: A parallel accelerator simulation code for electron generation and tracking, AIP Conf. Proc. 877, 241 (2006). 